This is the peer reviewed version of the following article: Wright, C. (2016), AssessmentSensitivity: The Manifestation Challenge. Philosophy and Phenomenological Research, 92: 189-196. doi: 10.1111/phpr.12262, which has been published in final form at http://onlinelibrary.wiley.com/doi/10.1111/phpr.12262/full. This article may be used for noncommercial purposes in accordance With Wiley Terms and Conditions for self-archiving. 
For a P\&PR Book Symposium on John MacFarlane's Assessment-Sensitivity: Relative Truth and Its Applications

\title{
Assessment-Sensitivity: the Manifestation Challenge
}

\author{
Crispin Wright
}

I

MacFarlane's core project in his deep-reaching, superbly crafted book is the defence of the claim that there is a theoretically respectable, interesting, useful species of relativism about truth—the species he captions by the term, assessment-sensitivity. Assessmentsensitivity contrasts, however, with classic truth relativism - if indeed there is such a thing - in three important respects. First, it is potentially a local feature of discourses. MacFarlane, unlike Protagoras, is making no general claim about the metaphysics of truth. So he finesses a broad sweep of traditional concerns about the coherence of truthrelativism, from the Theaetetus onwards, which take it to be a global thesis, (so hence, e.g., self-applicable.) Second, whereas traditional relative truth is a property of the contents of attitudes, assessment-sensitivity is a characteristic in the first instance of token assertoric utterances - though MacFarlane allows it to apply derivatively to the propositions expressed thereby (which he understands in the usual intuitive way as what are asserted, what are believed, what sustain relations of incompatibility and entailment, and so on. ${ }^{1}$ ) Finally, MacFarlane's project is harnessed to the task of giving descriptively adequate semantic theories for certain regions of discourse as actually practiced, rather than, at least in the first instance, to any specifically metaphysical controversies. Traditionally,

${ }^{1}$ See Assessment-Sensitivity p. 49 and following. Unless otherwise stated, all references below are to MacFarlane's book. 
truth-relativism is a player in the normative debates about realism and objectivity, one kind of paradigm of anti-realism, alongside and contrasting with non-cognitivism, errortheory, expressivism and the rest, - and indeed a paradigm that in the modern (20th century) debates was largely discarded. MacFarlane, as it appears, intends no direct contribution to those debates.

The interest of MacFarlane's thesis is nevertheless unquestionable. The remarks to follow will bear on the theoretical respectability, broadly conceived, of truth-relativism as he develops it, and —indirectly—on its utility.

II

A class of utterances is assessment sensitive if and only if, for each member $U$ of that class, the question whether an assertor of $U$ speaks truly turns not merely on the circumstances obtaining on the occasion of the assertion but depends additionally on aspects of a 'context of assessment': perhaps the information state, or interests, of an assessor, or her moral standards, or tastes, or just the time of assessment. A single such utterance may thus receive variable but nevertheless correct assessments of truth-value, depending on the variable characteristics, broadly conceived, - 'parameters' - of assessors of it. There is no once-and-for-all answer to the question whether the assertor of $U$ spoke truly, even after the content and circumstances of the assertion are fully determined. Thus the truthconditions for assessment-sensitive utterances involve a double relativity: $U$ is true (or not) as uttered in $\mathrm{C}_{1}$ - the context of use, serving to fix the content expressed by $\mathrm{U}$ (as well, perhaps, as other relevant features of the circumstances of evaluation) - and 
assessed in $\mathrm{C}_{2}$ - the context of assessment, serving to fix the values of the relevant parameters of assessment. ${ }^{2}$

Now, if a semantic theory that centralises such an assessment-sensitive notion of truth is to prove descriptively superior, we need a clear account of how the assessmentsensitivity of a class of utterances might be distinctively manifest in the discourse concerned. What is wanted, at first blush, is clear evidence that a pair of assessors who diverge in their assessments of an utterance $\mathrm{U}$, one regarding it as true and the other as false, can both be not merely justified but correct- and this purely in virtue of their satisfaction of different relevant parameters of assessment. How might that highly theoretical-sounding circumstance show in linguistic practice?

I think this matter has always been problematic, and my principal point here will be that it remains so. Of course there would be no problem if we could just assume that each speaker $\mathrm{X}$ has the resource in the object language of a correctness predicate, contrasted with all three of (absolute) truth, truth as assessed by $\mathrm{X}$, and epistemic justifiability in the object language. In that case X can simply say that Y's assessment is correct, even though false (as assessed by X), and even though X does not mean to say (merely) that Y's assessment is fully (epistemically) justified in Y's situation. But the theorist of assessment-sensitivity cannot simply assume that such resources are in play. We are asking after the pre-theoretic manifestation of the fact that the discourse is assessment-sensitive: we want to be told about patterns of use that will display that fact even if the only alethic predicate in currency in the discourse itself is the unreconstructed

\footnotetext{
${ }^{2}$ The extension of this idea to propositions is outlined in chapter 4.
} 
"true". The task is to give sense to the idea that a semantic theory featuring an assessment-sensitive notion of truth has genuine, distinctive operational content.

\section{III}

MacFarlane himself is very respectful of the manifestation-challenge. His response to it is fashioned in reaction to another type of proposal that comes into focus in his framework- one, indeed, which may seem to chime better with certain folk ideas about relativism than MacFarlane's own. Suppose Tim affirms, correctly by his taste, that stewed rhubarb is delicious, and Paul affirms, correctly by his taste, the contrary. And suppose I want to say, even-handedly, as an expression of an intuitive relativism, that neither is making any kind of mistake. (Their disagreement is "faultless".) That is something it seems I cannot say if utterances of "Stewed rhubarb is delicious" are assessment-sensitive, since then I will have to assess both claims by my taste, and whatever that may be, it cannot sustain both Tim's and Paul's verdicts. Suppose therefore that we retain the idea that the truth of such assertions is standards-of-taste-relative but stipulate instead that the standards by which they are to be assessed are those of their authors. The proposal is, in effect, that $\mathrm{C}_{1}$ and $\mathrm{C}_{2}$ are to coincide: the context of assessment is to be the context of use. Then now I can say that both Paul and Tim speak truly.

This proposal is what MacFarlane calls non-indexical contextualism. ${ }^{3}$ Non-indexical contextualism may seem at first glance like a pretty decent approximation to Everyman's

\footnotetext{
3 "Non-indexical" indicates that the view does not postulate that the propositional content of "Rhubarb is delicious", e.g., varies as a function of variation in parameters-here, standards of taste-in Tim's and Paul's respective contexts of use. What Tim affirms is what Paul denies. But the view is contextualist in granting that the truth-value of the proposition concerned is variable with parameters set by context of use.
} 
notion of relativism. (It allows that there is "your truth" and "my truth".) MacFarlane scorns the idea that such a view is properly relativist, primarily on the ground that, unlike assessment-sensitivity, it assigns once-and-for-all truth-values to Paul's and Tim's utterances. That reflection, notably, misses the point that if Paul insincerely but obsequiously defers to Tim, one and the same proposition will be false in his mouth but true in Tim's. The reader may think that is relativism enough to fulfil the Protagorean spirit.

Perhaps it is pointless to debate further which view has the better title to the term, "relativism". What is significant for MacFarlane's purposes is that non-indexical contextualism and assessment-sensitivity potentially diverge in their predictions of use. For suppose I assert U and then later have occasion to re-visit the question of the truthvalue of my former utterance of it: non-indexical contextualism bids me defer to the standards of the context in which the utterance was originally made, and if it was correct by those standards, - let's suppose it was, - I should now regard my assertion as having been made truly, whatever the standards of the context that I now occupy. Assessmentsensitivity, by contrast, will have me assess my earlier utterance of $U$ precisely by the standards of my present context and I may thus be led to regard my assertion as having been made falsely, even if it was correct by the standards of its original context.

\section{IV}

This contrast is key to MacFarlane's response to the manifestation-challenge. He approaches the matter via a remark of Michael Dummett in the latter's paper, "Truth", to the effect that in order to grasp the notion of truth, it is necessary not merely to know 
under what circumstances sentences are true, but also to understand the connection between their truth and the proprieties of their assertion. ${ }^{4}$ MacFarlane casts this as the claim that assertion is constitutively governed by the following

Truth Rule: At a context $\mathrm{C}$, assert that $\mathrm{P}$ only if $\mathrm{P}$ is true at $\mathrm{C} .{ }^{5}$

We presumably know well enough what it is for speakers to manage their linguistic practice in conformity to the Truth Rule. Correspondingly, it ought to suffice to allay qualms about the theoretical respectability of assessment-sensitive truth to formulate a corresponding rule for it—a rule such that it is clear what it would be to manage a relevant linguistic practice in conformity to it - and maybe thereby to show what would be for practitioners to have implicit grasp of assessment-sensitive truth. ${ }^{6}$

MacFarlane, however, fails to find any suitable such rule distinctive of assessmentsensitive truth. He discusses various options and finds all wanting, ${ }^{7}$ with the best candidate being:

${ }^{4}$ Page 98 and following.

${ }^{5}$ Page 101. In speaking of this rule as constitutive, MacFarlane intends, I imagine, not that an utterance is an assertion if and only if compliant with it, nor if and only if the utterer intends so to comply, but that it is of the essence of assertion to be answerable to it - to be subject to negative appraisal, just on that account, if in breach of it. Cf. note 7 on page 101.

6 This, to be sure, will only address the question of theoretical respectability narrowly conceived: the question of giving sense to the theoretical primitive. The broader project is that of rendering fully intelligible a practice for whose description the notion of assessment-sensitivity is needed. For this, the relativist needs not merely to characterise the outlines of a practice in which the operation of assessment-sensitivity would be distinctively manifest but to show how such a practice could be rational and useful. MacFarlane encroaches on this vital task in chapter 12 - but I have no space to consider his suggestions here.

7 Pages 103-4. 
Reflexive Truth Rule An agent is permitted to assert that $\mathrm{P}$ at context $\mathrm{C} 1$ only if $\mathrm{P}$ is true as used at $\mathrm{C}_{1}$ and assessed from $\mathrm{C}_{1}$

— and the evident problem with this is that since it identifies the contexts of use and assessment, it predicts nothing that is not predicted by non-indexical contextualism. In fact it is clear that no rules for assertion alone can do what's wanted, since rules for assertion - at least, rules which agents can effectively follow- have to relate to features of prospective contexts of use, and so can make no room for an active contrast between context of use and context of assessment.

MacFarlane's solution is to invoke the idea of retraction. The assessmentsensitivity of an utterance will show, MacFarlane contends, in its being constitutively subject to a norm of retraction along the following lines:

Retraction Rule. An agent in context $\mathrm{C}_{2}$ is required to retract an (unretracted) assertion of $\mathrm{P}$ made at $\mathrm{C}_{1}$ if $\mathrm{P}$ is not true as used at $\mathrm{C}_{1}$ and assessed from $\mathrm{C}_{2}$

Non-indexical contextualism need have no quarrel with the Retraction Rule as such. But, unlike assessment-sensitivity, it will require an assessor at $\mathrm{C} 2$ to return the same verdict as a correct assessment from the standpoint at $\mathrm{C} 1$ and so will mandate retraction only if the assertion was not correct by the lights of the latter. So the two views will thus potentially diverge in their predictions of retraction.

\section{V}

Retraction phenomena are thus a crucial component in the empirical distinguishability of MacFarlane's proposal from non-indexical contextualism. They are also key to MacFarlane's main strategy of argument against regular-indexical-contextualism. 
Regular contextualists about "knows", for example, make much of the apparent variation in the assertibility of knowledge ascriptions in the light of switches between so-called "low" and "high" contexts. However when, in the scepticism seminar, I decline to ascribe knowledge to a subject— say, myself — to which earlier over breakfast I laid claim, I will characteristically deny that I spoke truly in making that claim, saying things like "I realise now that I didn't know anything of the kind". Yet on the face of it, standardscontextualism about "knows" - whether indexical or non-indexical - can explain only my denial that I know now; it should predict no quarrel with what I said earlier, since the relevant form of words has undergone a shift in content with the tightening of the standards. ${ }^{8}$ It is similar with epistemic "mights" and "coulds". High up on the Cuillin ridge during a misty scramble, a large peak briefly looms into view seemingly just a short way ahead. "That could be the summit", you encouragingly say. Later, warming your feet by the fire and poring over the map, you say, "You know, I was wrong. That peak couldn't have been the summit. It's not visible from where we were standing." That seems like an acceptable piece of conversation, in which you correct-retract- an earlier claim which, we can suppose, was proper enough in the original context of use. For MacFarlane, it is the task of accounting for data of correction and retraction of this kind that provides the principal raison d'être for relativism. Since no form of contextualism can, apparently, account for such data, assessment-sensitivity is the superior theory.

8 That I regard my former assertion as false is the crucial point in this instance; in view of the factivity of all (serious) uses of "knows", contextualism can of course predict that I will no longer wish to endorse the truth of my earlier utterance. What it seems it cannot predict is my denial that what I said is true. 


\section{VI}

Does this address the manifestation-challenge? Let us grant that if the issue was only whether we should prefer a (regular) contextualist or an assessment-sensitivity relativist account of a given region of discourse, then differences in retraction behaviour may well favour the latter. Caution is, admittedly, needed with the point, for it depends on how exactly the notion of retraction is understood and how it in turn is manifest in linguistic practice. Generally, MacFarlane characterises retraction as an operation on speech-acts: it is the withdrawal of an assertion, comparable to the withdrawal of a question, the cancellation of a command, or the revoking of a promise. ${ }^{9}$ There is an issue, which I cannot adequately enter into here, about whether this notion of retraction-contrast: denial of the former assertion- will underwrite the problem MacFarlane thinks it makes for contextualism in all the relevant cases. Generally, one will regard a previous assertion as retractable in this milder sense whenever considerations emerge which undermine ${ }^{10}$ the original grounds for it - and this is so whether or not the content of the utterance in question was sensitive the context of use. The consequent worry is that some at least of the retraction patterns that seem characteristic of our use of e.g. epistemic modals may accordingly be readily explicable by contextualism. This is plausibly so, for example, in the Cuillin peak example: the evidential relevance of the appearance of the false summit

9 Page 108.

10 I have in mind the usual contrast between undermining and overriding defeaters. 
through the mist is undermined by the later information that the relevant peak was not visible from that location. ${ }^{11}$

However whether the intuitions that MacFarlane tries to excite concerning retractions in some of his case studies really do provide for crucial experiments between assessment-sensitivity and contextualism is not an issue I can take further here. The issue I wish to press is whether enough has been done to have assessment-sensitive truth make clear theoretical sense in the first place. MacFarlane writes that "What makes the relative truth intelligible is the potential difference between the context at which an assertion is made and the contexts at which challenges to it will have to be met and retractions considered."12 But he knows very well that only that much does not make relative truth intelligible, since that potential difference will engage any non-relativistic discourse where shifting evidential states can mandate the retraction of previously warranted claims.

If we are already persuaded of the intelligibility of relative truth, we can characterise the difference between the two kinds case like this: when new evidence comes in that potentially mandates retraction of a non-relativistic assertion that was warranted in its original context, the justifiability of the retraction will require grounds for thinking that the original warrant was misleading. In a relativistic case, however, so one would suppose, there should be no such requirement. That is: if a practice is to distinctively manifest a relativistic character, then something about the way that retraction of an assertion is done will need to evince not merely that the assertion is

${ }^{11}$ Contrast the Chainsaw example in Wright (2007)

12 Page 116. 
acknowledged to have been fully justified its original context, but that there need have been nothing misleading about the support it then had - that it was, as it were, alethically faultless back in that context. But the question we keep coming back to is: how might ordinary object-level practice manifest that element?

My sense is that MacFarlane underestimates the difficulties that his proposal encounters at this point because he envisions a theoretical landscape in which there are essentially just three players: assessment-sensitivity, one or another form of contextualism, and invariantist realism. The latter will expectably also predict the patterns of retraction behaviour that McFarland believes favour assessment-sensitivity. The reason why he thinks that assessment-sensitivity is prioritised by retraction phenomena is because he has already discounted realism in the relevant cases. There is thus a metaphysical lemma required by his strategy of argument.

It may be rejoined that, for the relevant range of cases, that is surely a point of no great moment. There is no major metaphysical hostage involved, surely, in the rejection of realism about basic taste, comedy, or epistemic possibility (though the issue is of course much more controversial when it comes to the case of knowledge.) The real concern, though, is not about the warrant for discounting a third possibility-realismbut about that for discounting a fourth: minimalism.

I can here offer only the briefest outline of this direction. For the minimalist, the object-language truth-predicate that features in the discourses in which we are interested is, more or less, a deflationary-merely disquotational—truth-predicate. And with that, as he notes, MacFarlane can agree. ${ }^{13}$ But minimalism rejects the idea that any

13 Pages 38-9 and 93-4. 
special notion of truth is required in framing a semantic theory for these discourses. With that, again, MacFarlane can agree provided the interest of the theorist is merely in the mechanisms of semantic composition. But if the project is to recover and make sense of (diachronic) patterns of assent and dissent, MacFarlane will disagree; it is here that the invocation of assessment-sensitivity is supposed to come into its own. Minimalism disputes that. For the minimalist, understanding the practice of these discourses is a matter of understanding the conditions under which their signature statements may be asserted, and the conditions under which such assertions are defeated and should be withdrawn or denied. The former are typically subjective states: states of information, or various kinds of affective state. The defeaters are various and variously topic-specific but, to illustrate for the case of taste, will include evidence of impairment of appropriate capacities (You just cleaned your teeth, or are still suffering the effects of dental anaesthesia), evidence of lack of community ("No-one but you likes that stuff"), overriders drawing on superordinate values ("Do you have any idea how they treat the geese?") and evidence of instability in one's relevant responses. It is the role of such defeaters that gives sense to the contrast between the 'objectified' idiom: “This paté is delicious" and the mere report of one's personal affect :"I like this paté" which share their assertibility conditions but differ in their conditions of retraction - both withdrawal and denial. The general tenor of the defeaters in the case of taste is to register our interest in stable, shareable, (morally) acceptable gustatory responses.

The foregoing is just an illustration, and a sketchy one at that. But perhaps it is enough to give focus to the challenge I want to table. Suppose we are given a refined and 
elaborated description of the conditions of assertion and defeat that are characteristic of the signature claims of one of the discourses for which MacFarlane is proposing an assessment-sensitive semantics. Suppose we are also given a plausible informal theory of the social purposes characteristically served by a discourse that is so patterned. And suppose finally that we have an orthodox compositional semantic theory for the discourse . Then what further legitimate explanatory ambition is there, so far unaddressed, which a semantic theory based on assessment-sensitivity might show its superiority by accomplishing?

It may be helpful to sketch the contrast, in the most general terms, between the relativist and minimalist approaches to disagreements in basic taste. If the intuitive idea that such disagreements can somehow be faultless is not to reduce to the banality that the respective opinions may be fully justified, then the intention of the idea has to be that they may somehow reflect no alethic fault. This is, naturally, a hopeless idea if the discourse is thought of as answerable to a single norm of truth with which no statement and its negation can simultaneously comply. So if faultless disagreement is to be a possibility, there must be no such single alethic norm. That leaves two options. One is, in one way or another, to-as it were-fracture the norm, multiply the ways of being true and spread the pieces around, so that conflicting opinions can each alight on a shard. Any relativistic account of faultless disagreement offers a particular implementation of that option. The other option is to suction out the substance of the alethic norm, leaving only the deflated residue, and look elsewhere for the substantive norms that operate over the discourseto look, as Wittgenstein urged, at the use. I regard it as a strength of the combination of propositional minimalism and alethic pluralism defended in Truth and Objectivity that it 
provides a natural setting for the elaboration of the second direction, which I hope to return to in further work. ${ }^{14}$

New York University and University of Stirling

Additional references

Dummett, Michael (1958) "Truth", Proceedings of the Aristotelian Society, Supplementary Volume LIX, 141-62

Wright, Crispin (1992) Truth and Objectivity, Cambridge, Mass: Harvard University Press

(2007) "New Age Relativism And Epistemic Possibility: the Question of Evidence", Philosophical Issues 17, 262-283

${ }^{14}$ I would like to thank Filippo Ferrari for helpful comments on an earlier draft and John MacFarlane for valuable advice. 